malperfusion of the celiac trunk, superior mesenteric artery, renal arteries, and left external iliac artery.

Emergency endovascular stent grafting was performed 6 hours after the initial operation. Two aortic selfexpendable, noncovered stents $(\mathrm{E}-\mathrm{XL}, 32 \mathrm{~mm} \times 130$ $\mathrm{mm}$ and $28 \mathrm{~mm} \times 100 \mathrm{~mm}$; Jotec $\mathrm{GmbH}$ ) were placed in the dissected thoracoabdominal aorta to expand the true lumen and increase perfusion of the abdominal branches. Persistent superior mesenteric artery malperfusion was treated with 2 balloon-expandable Herculink stents $(6.5 \mathrm{~mm} \times 18 \mathrm{~mm}$; Guidant, Indianapolis, Ind), and 2 self-expandable nitinol SMART iliac stents (8 $\mathrm{mm} \times 60 \mathrm{~mm}$ and $7 \mathrm{~mm} \times 40 \mathrm{~mm}$; Cordis Corperation, Bridgewater, NJ) were deployed into the left external iliac artery. Postprocedure angiography showed sufficient perfusion of all abdominal aortic branches and the arteries of the left leg (Figure 1).

The patient was successfully weaned from the ventilator 8 weeks postoperatively after a prolonged clinical course. Eleven weeks after the initial operation, she was mobilizing fully without any permanent neurologic deficits.

\section{DISCUSSION}

The frozen elephant trunk technique allows for singlestage repair of the ascending aorta and transverse aortic arch after antegrade endovascular treatment of the DA. ${ }^{1,2}$ In patients with acute type A dissection, coverage of additional intimal tears and improved true lumen perfusion may lead to false lumen exclusion and obliteration after the frozen elephant trunk procedure. ${ }^{3} \mathrm{Al}-$ though midterm results for this technique are promising, controversy exists as to whether a hybrid or a conventional approach should be applied for type A aortic dissection. . $^{3,4}$

The case described herein reveals an unexpected intraoperative complication requiring switching from a hybrid to a conventional surgical repair with extra-anatomic reconstruction of the thoracic aorta, followed by emergency endovascular treatment of the remaining aorta owing to significant malperfusion. Our case illustrates the complex and multidisciplinary approach that is occasionally required for patients with acute aortic dissection.

The precise cause of the technical device failure during our frozen elephant trunk operation is difficult to determine. We can postulate, however, that stent-graft deployment in the very small true lumen might have resulted in trapping of the device's nose cone and inability to extract the prosthetic portion of the hybrid device. A newer version of the E-vita stent graft (E-vita open plus; Jotec $\mathrm{GmbH}$ ) now has a much lower profile nose cone to prevent this problem from occurring.

\section{References}

1. Karck M, Chavan A, Hagl C, Friedrich H, Galanski M, Haverich A. The frozen elephant trunk technique: a new treatment for thoracic aortic aneurysms. J Thorac Cardiovasc Surg. 2003;125:1550-3.

2. Tsagakis K, Pacini D, Di Bartolomeo R, Benedik J, Cerny S, Gorlitzer M, et al. Arch replacement and downstream stent grafting in complex aortic dissection: first results of an international registry. Eur J Cardiothorac Surg. 2011;39:87-93; discussion 93-4.

3. Uchida N, Ishihara H, Shibamura H, Kyo Y, Ozawa M. Midterm results of extensive primary repair of the thoracic aorta by means of total arch replacement with open stent graft placement for an acute type A aortic dissection. J Thorac Cardiovasc Surg. 2006;131:862-7.

4. Dobrilovic N, Elefteriades JA. Stenting the descending aorta during repair of type A dissection: technology looking for an application? J Thorac Cardiovasc Surg. 2006;131:777-8.

\title{
Long-term systemic right ventricular support in transposition and dextrocardia
}

\author{
Aekarach Ariyachaipanich, MD, ${ }^{\mathrm{a}}$ James O. Mudd, MD, ${ }^{\mathrm{a}}$ Jill Gelow, MD, ${ }^{\text {a }}$ and Howard K. Song, MD, PhD, ${ }^{\mathrm{b}}$ \\ Portland, Oregon
}

\footnotetext{
From the Divisions of Cardiovascular Medicine ${ }^{\mathrm{a}}$ and Cardiothoracic Surgery, Oregon Health \& Science University, Portland, Ore.

Disclosures: Authors have nothing to disclose with regard to commercial support.

Received for publication April 19, 2012; revisions received June 8, 2012; accepted for publication July 10, 2012; available ahead of print Aug 6, 2012.

Address for reprints: Aekarach Ariyachaipanich, MD, Oregon Health \& Science University, Mail Code UHN-62, 3181 SW Sam Jackson Park Rd, Portland, OR 97239 (E-mail: ariyacha@ohsu.edu).

J Thorac Cardiovasc Surg 2012;144:e108-10

$0022-5223 / \$ 36.00$

Copyright (C) 2012 by The American Association for Thoracic Surgery

http://dx.doi.org/10.1016/j.jtcvs.2012.07.017
}

For patients with complex congenital heart disease (CHD) who are in end-stage heart failure, a significant disparity in the use of mechanical circulatory support persists. ${ }^{1}$ Recent reports have shown the feasibility and initial success of supporting patients with systemic morphologic right ventricles (RVs) with continuous-flow ventricular assist devices (VADs) ${ }^{2}$; however, long-term outcome data are lacking. We present our successful experience with a HeartMate II left ventricular assist system (Thoratec Corporation; Pleasanton, Calif) in a mirror image position to support the 

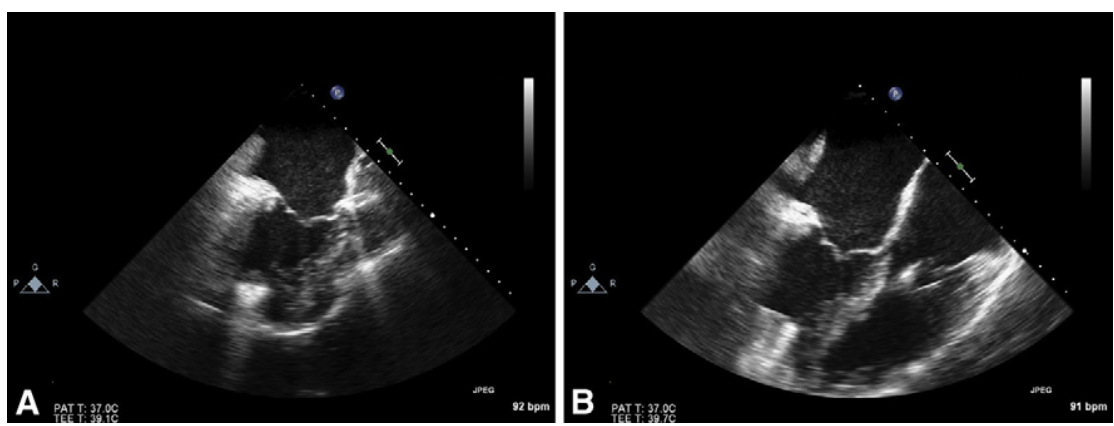

FIGURE 1. Intraoperative transesophageal echocardiography. A, The systemic right ventricular apex was indented by the surgeon to locate the appropriate location for the ventricular assist device inflow cannula. B, Appropriate positioning of the inflow cannula was confirmed after the procedure.

systemic morphologic RV in a patient with congenitally corrected transposition of the great arteries and situs inversus with dextrocardia.

\section{CLINICAL SUMMARY}

A 56-year-old man with a history of situs inversus, dextrocardia, atrioventricular discordance, and ventriculoarterial discordance (levotransposition of the great arteries) with a ventricular septal defect, atrial septal defect, and pulmonary stenosis was seen with progressive heart failure symptoms, systemic RV failure (ejection fraction $<20 \%$ ), and maximal oxygen consumption of $10.7 \mathrm{~mL} /(\mathrm{kg} \cdot \mathrm{min})$, despite both optimal medical therapy and biventricular pacing. This patient had undergone 2 previous sternotomies for atrial and ventricular septal defect repair and pulmonary valvotomy at 13 and 31 years of age, respectively. He was referred for a heart transplant; however, he was found to have renal cell carcinoma, which was treated by nephrectomy. VAD implantation was therefore recommended for long-term support to ensure cancer-free survival.

Preoperative imaging for the implantation of a HeartMate II VAD confirmed the systemic ventricle to be a morphologic RV that was located posterolaterally to the morphologic left ventricle in the right hemithorax. A reoperative median sternotomy was performed. Exposure of the patient's aorta was limited by its rightward displacement and the large overlying pulmonary artery. Cardiopulmonary bypass was instituted from the left femoral vessels. The VAD outflow graft was running to the left (medial) of the heart and was anastomosed to the anterior ascending aorta.

The surface of the RV apex was indented and observed on intraoperative transesophageal echocardiography to select an appropriate cannulation site (Figure 1, $A$ ). Prominent trabeculae were encountered and were aggressively resected in the area of the ventriculotomy to prevent obstruction of the inflow cannula. The VAD inflow cannula was then implanted in the standard fashion, VAD support was initiated, and the patient was weaned from cardiopulmonary bypass.

Transesophageal echocardiography was also used to confirm appropriate orientation of the VAD inflow cannula toward the atrioventricular valve (Figure $1, B$ ). Because of the posterolateral displacement of the systemic RV and the posterior direction of the apex, the VAD pump and inflow cannula needed to be aligned in a nearly anteroposterior direction to maintain the proper orientation (Figure 2).

The patient had an uncomplicated postoperative course and was discharged 13 days after the operation. He remains free of symptoms and has not required readmission to the hospital in more than 1 year.

\section{DISCUSSION}

Because of advances in pediatric cardiovascular care, most children with complex CHD survive to adulthood. Among adults with CHD, ventricular dysfunction and heart failure are especially common in those with single or

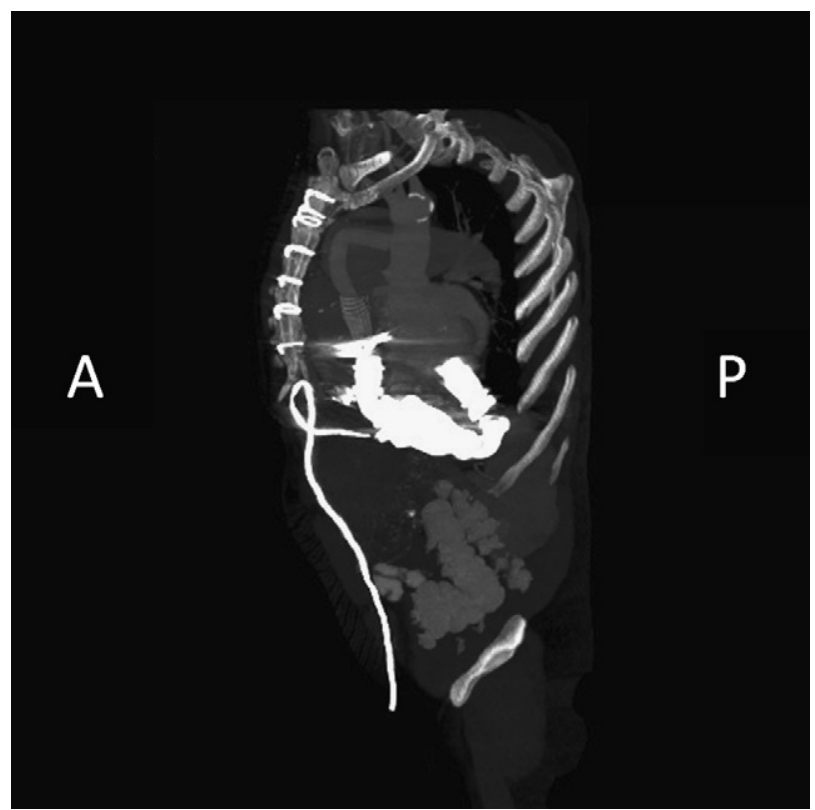

FIGURE 2. Postoperative sagittal computed tomographic scan reconstruction demonstrating the near anteroposterior orientation of the ventricular assist device pump and inflow cannula within the right hemithorax. $A$, Anterior; $P$, posterior. 
systemic RV, and such patients represent a growing subset of the population being listed for heart transplants. The need for mechanical circulatory support for this patient population is therefore growing.

Familiarity with CHD anatomy, morphology, and variations in the hemodynamic physiology is important when implantation of a VAD is being contemplated. For example, the $\mathrm{RV}$ is more triangular and has prominent trabeculations and the moderator band near the apex. All these characteristics may affect flow into the VAD inflow cannula and lead to low flow, suction events, or VAD obstruction and thrombosis. Attention to the potential need for resection of the trabeculae and the moderator band is important. There have been reports of the use of both pulsatile and continuousflow $\mathrm{VADs}^{2}$ in morphologic RVs to support the systemic circulation. Preoperative and intraoperative imaging is critical in achieving optimal VAD inflow cannula orientation.

Although the HeartMate II VAD is not designed for patients with dextrocardia, it can be adapted to them. ${ }^{3}$ When the device is rotated $180^{\circ}$, its orientation is reversed, making it appropriate for implantation into the right hemithorax. Next-generation VADs with smaller pump designs will likely minimize this issue.

This case illustrates how patients with complex CHD lesions can be supported with VADs with good long-term outcome as long as proper attention is paid to anatomic details and their potential impact on device implantation and function. Further studies are necessary to confirm the applicability of this technology across the spectrum of CHD.

\section{References}

1. Everitt MD, Donaldson AE, Stehlik J, Kaza AK, Budge D, Alharethi R, et al. Would access to device therapies improve transplant outcomes for adults with congenital heart disease? Analysis of the United Network for Organ Sharing (UNOS). J Heart Lung Transplant. 2011;30:395-401.

2. Joyce DL, Crow SS, John R, St Louis JD, Braunlin EA, Pyles LA, et al. Mechanical circulatory support in patients with heart failure secondary to transposition of the great arteries. J Heart Lung Transplant. 2010;29:1302-5.

3. Faggian G, Forni A, Luciani GB. LVAD in situs viscerum inversus totalis. J Heart Lung Transplant. 2011;30:1420-1. 